\title{
Value of High Frequency Sonography in Rheumatoid Arthritis
}

\author{
Andrea S. Klauser ${ }^{1}$, Mohamed A. Bedewi ${ }^{2}$, Mihra S. Taljanovic ${ }^{3}$, Johann Gruber ${ }^{4}$, \\ Silvia Strobl ${ }^{1}$, Thomas Auer ${ }^{1}$, Stefan Rauch ${ }^{1}$, Erich Mur $^{3}$, Werner R. Jaschke ${ }^{1}$ \\ Departments of Diagnostic Radiology ${ }^{1}$, Medical University Innsbruck, Austria; \\ Radiology ${ }^{2}$, Salman Bin Abdulaziz University, Kingdom of Saudi Arabia \\ Medical Imaging ${ }^{3}$, The University of Arizona Health Network, \\ Internal Medicine ${ }^{4}$, Medical University Innsbruck, Austria
}

\begin{abstract}
Rheumatoid arthritis is a chronic inflammatory arthritis with the incidence reaching up to $1 \%$ of the population. The treatment of rheumatoid arthritis (RA) has improved dramatically over the last years with the introduction of TNF (tumor necrosis factor) alpha inhibitors. Joint damage and functional impairment are highly important adverse outcomes of rheumatoid arthritis. They have been repeatedly shown to be associated with clinical disease activity, in particular with swollen joint counts and acute-phase reactant levels, as well as composite measures of disease activity in which these variables are included as components. OMERACT outcome measures in rheumatology group published consensus US definitions for common pathologic lesions observed in patients affected by RA, each of them is discussed, effusion, synovitis, bone erosion, hypervascularity and tenosynovitis. The use of musculoskeletal ultrasound (MSK-US)is growing more and more between radiologists and rheumatologists for the early diagnosis and therapeutic follow up of rheumatoid arthritis and US scores are used for monitoring RA disease activity; US findings can be scored using quantitative or semiquantitative scoring systems to estimate the degree of synovial/tenosynovial and erosive processes.. New technologies such as elastography, three dimensional sonography, fusion imaging and contrast enhanced ultrasound can add further powerful information in the imaging of RA. [Egypt J Rheumatology \& Clinical Immunology, 2015; 3(1): 23-32]
\end{abstract}

Key Words: Sonography; Rheumatoid Arthritis; erosions;synovitis, erosion

\section{INTRODUGTION}

Rheumatoid arthritis (RA) is a progressive systemic chronic inflammatory disease that affects primarily the synovial membrane and can lead to bone and cartilaginous destruction ${ }^{1}$.

The increasing emphasis on the early diagnosis and monitoring of this condition has led to the greater involvement of advanced imaging techniques such as ultrasound (US) and MRI ${ }^{2}$.

Early detection and accurate assessment of RA activity in a non-active state is of the utmost importance because therapeutic decisions should target sustained remission or, at a minimum, the lowest possible disease activity to improve RA outcomes ${ }^{3}$.

Musculoskeletal (MS) ultrasound (US) has been widely employed in the assessment and monitoring of rheumatic diseases, particularly in patients affected by RA. Thanks to its characteristic of validity, reliability, reproducibility and sensitivity to change, US superseded other commonly used imaging modalities, such as plain radiography (X-ray), and its use has been included in the daily routine clinical practice in rheumatology ${ }^{4}$.

For Correspondence: Email: mohamedbedewi@yahoo.com
In the current paradigm for management of patients with rheumatoid arthritis (RA), obtaining clinical remission of symptoms remains the most important aim, but achieving radiographic remission is another key goal of treatment. Several parameters detectable by musculoskeletal ultrasonography can predict the development of severe RA, as well as monitor patients' responses to treatment; thus, musculoskeletal ultrasonography is widely used for evaluating patients with RA, both in clinical trials and in clinical practice ${ }^{5}$.

The use of powerful and expensive biologicals (TNF (tumor necrosis factor) alpha inhibitors) has radically changed the outlook for patients with RA, aiming prevention of irreversible joint damage and the consequent symptoms and deformities associated with it ${ }^{2}$.

The exceptional representation of acute inflammatory soft tissue processes, very early recognition of bony destruction and the ubiquitous availability of the method have been major contributors to this success. In recent years there have been new developments in technology and in examination methods. The substantial importance of sonography for early detection of arthritis, differential diagnostics, therapy monitoring and estimation of prognosis is underlined by the continuously increasing number of international publications. Several scoring systems have been developed for small and large 
joints and have been proven not only under study conditions but also in practice ${ }^{6}$.

Subclinical inflammatory processes which are held responsible for the so-called silent progression can be detected using sonography ${ }^{6}$.

The great advantage and prestige of the ultrasonographic study, which has motivated enthusiastic research in the area, resides in its capacity to detect synovitis and bone erosion at a preradiographic phase. That generates information that can be used for diagnostic or therapeutic purposes, with a potential impact on the patients' quality of life ${ }^{7}$.

Joint damage and functional impairment are highly important adverse outcomes of (RA). They have been repeatedly shown to be associated with clinical disease activity, in particular with swollen joint counts and acutephase reactant levels, as well as composite measures of disease activity in which these variables are included as components (1-3). Moreover, in states of very low disease activity, progression of joint damage is related to residual local joint inflammation rather than acute-phase reactant levels ${ }^{2}$, and the association between progression and joint swelling has been observed at the level of individual joints. Today's therapeutic targets in patients with RA are achievement of disease remission or low disease activity, whereby the term remission comprises lack of clinical disease activity. It has been suggested that some patients may experience radiographic progression of joint disease despite being in clinical remission ${ }^{8}$. Therefore true remission, visualized by using Power Doppler Ultrasound (PDUS) with a decrease of intraarticular hypervascularity might contribute to stop joint destruction.

Standardised multiplanar scans have been developed for each joint region according to the guidelines of the European League Against Rheumatism (EULAR). Therefore, standardized complete joint scanning is recommended ${ }^{9}$. In 2001 the EULAR guidelines indicated the standard scans to perform at each joint for the evaluation of articular and peri-articular structures (eular), these recommendations were reinforced by the treat-totarget approach with remission set as the primary treatment goal for RA in everyday clinical practice ${ }^{10}$. In 2005, the OMERACT group published consensus US definitions for common pathologic lesions observed in patients affected by RA, each definition will be mentioned with the appropriate sign ${ }^{4}$.

Musculoskeletal US scoring systems are used to verify clinically detected RA disease activity and to assess therapeutic response. Several musculoskeletal US scores are used for monitoring RA disease activity; US findings can be scored using quantitative $(\mathrm{mm})$ or semiquantitative $(0-3)$ scoring systems to estimate the degree of synovial/tenosynovial and erosive processes. Additionally, US findings can be described on a binary (1/0) basis. International/ European US expert groups are working towards the development of a standardised US scoring system which will reflect a patient's global disease activity. A scoring system for synovitis in RA which combines gray scale findings (GSUS) (=B-mode) and PDUS findings in a semiquantitative $(0-3)$ grading system is in the process of development, but consensus about the joint regions and the optimal (minimal) number of joints has not yet been reached; US sum/composite scores of a reduced joint count reflect the overall RA disease activity in a short examination time (at a patient level) ${ }^{11}$.

A dynamic examination is necessary in order to detect small collections of fluid. The additional use of PDUS helps in differentiating active and inactive articular synovial/tenosynovial processes, especially in small joints ${ }^{11}$.

Current activities of the EULAR/OMERACT US task force include the development of a Global OMERACT Sonography Scoring (GLOSS) system in RA. Its feasibility, sensitivity to change and value over standard clinical care are being tested (data not yet published). GLOSS examines a number of small joints for synovitis, and the results evaluated by GSUS and PDUS are combined in this scoring system ${ }^{12}$.

\section{Tenosynovitis and Tendon Involvement}

Tenosynovitis can be one of the key features in patients with rheumatoid arthritis and longstanding tenosynovitis may result in tendon damage either by synovial proliferation or by bony attrition resulting in tendon rupture with consequent disability ${ }^{13}$.

Tenosynovitis is defined as hypoechoic or anechoic thickened tissue with or without fluid within the tendon sheath with possible signs of Doppler signals, which is seen in two perpendicular planes ${ }^{14}$. Both tendon disease and tenosynovitis are important features of RA, and US provides an ideal tool for their investigation. Tenosynovitis is seen as a combination of synovial thickening within the tendon sheath and tendon sheath effusion.

When this occurs close to a joint, an important role of US is to distinguish tenosynovitis from underlying joint disease. RA may bring about damage to the tendons themselves seen as either tearing or complete rupture. This occurs due to weakening of the tendons but also due to impingement of the tendons on adjacent bony structures. US is ideally suited for identifying the site of any tear and, if surgery is contemplated, assessing the length of the tear and site of the retracted ends. Associated abnormalities such as swan neck or boutonniere deformities may also be delineated on US to assess the tendon involvement. Early extensor tendon subluxation and luxation tendency because of extensive synovial proliferation at the level of the MCP joints can be well visualized by using dynamic US in order to prove stability of the sagittal bands. Another important soft tissue manifestation of RA is the development of 
rheumatoid nodules, reported in up to $30 \%$ of patients with RA; these lesions are usually found at sites of pressure such as the extensor aspect of the elbow, the heel, and the fingers. US demonstrates nodules as having a mixed echotexture, but they are generally hypoechoic and often contain fluid components and usually have poor internal vascularity ${ }^{2}$. Figure $1 \mathrm{~A}-\mathrm{D}$

\section{Synovium}

Normal synovium is not seen at ultrasound, but when thickened it is appreciated as abnormal intraarticular soft tissue that may, or may not, be associated with a joint effusion ${ }^{2}$.

Synovitis, either proliferative or exudative, is the earliest change that can be ultrasonographically graded. Its quantification via grayscale ultrasound usually uses a semiquantitative scale with three levels of intensity, indicating mild, moderate or marked synovial changes $^{15,16}$.

The term of synovitis is used to indicate the presence of synovial hypertrophy with PD signal and joint effusion. Changes in PD reflect modifications of disease activity, and the presence of PD has been associated with development of erosions ${ }^{17}$.

It is important to realize the potential pitfalls involved in Doppler ultrasound of synovitis, particularly issues relating to reproducibility, with factors such as probe pressure and variations in equipment being important in influencing reliability ${ }^{18,19}$. Therefore the probe should be held without tissue pressuring, when PD quantification is achieved.

According with the OMERACT indications, synovial fluid is defined as an abnormal hypoechoic or anechoic intra-articular material that is displaceable and compressible, and that does not exhibit Doppler signal $^{4}$. Figure $2 \mathrm{~A}-\mathrm{B}$

Recurrent episodes of tenosynovitis associated with proliferation of the tenosynovium can lead to tendon structural changes as tendon adhesion and rupture causing severe articular impairment ${ }^{20}$.

Palmar US examinations should accompany the dorsal examination both in clinical practice and in clinical trials ${ }^{21}$.

US-based disease activity estimation depends on the detection of synovitis. The most common synovitis abnormalities are proliferation, effusion, and neoangiogenesis. According to outcome measures in rheumatology clinical trials (OMERACT), synovial hypertrophy (proliferation) is defined as an abnormal hypoechoic intraarticular tissue that is nondisplaceable and poorly compressible, and which may exhibit Doppler signals because of hypervascularity, caused due to neoangiogenesis. Angiogenesis itself cannot be visualized by PDUS because of small vessel size (less than 40 micrometers), but US has the unique potential to demonstrate blood flow in angiogentic vessels by using contrast media, which are approved for clinical routine ${ }^{13}$.
Suggested scoring for PDUD are as follows: grade 0 : none; grade 1: minimal synovial thickening; grade 2 : synovial thickening bulging over the line linking tops of the periarticular bones without extension along the bone diaphysis; grade 3: synovial thickening bulging over the line linking tops of the periarticular bones with extension to at least one of the bone diaphyses.

\section{Bone Erosion}

Bone erosion results from the colagenase produced on the interface between synovium, bone and joint cartilage, typically observed in the periphery of the joint space, where bone is not covered by cartilage (=bare areas) ${ }^{22}$.

Erosions are appreciated on US as focal discontinuities in the bone cortex and have been defined on ultrasound as "a sharply marginated bone lesion, with correct juxta-articular location and typical signal characteristics, visible in at least two planes with a cortical break seen in at least one plane."15. Figure 1 A-D

The specificity of erosion detection on US is improved when only larger lesions with a size of 1-2 mm at least are considered, highlighting the recognized potential to confuse normal bone contours, surface irregularities for erosions and entering vasa nutritia. The need to demonstrate an erosion in two planes is mandatory for avoiding this problem. A familiarity with the normal osseous landmarks is also important to avoid this pitfall, although it is often useful to scan contralateral joints to further clarify any areas of confusion ${ }^{2}$.

Wakefield et al. described the first semiquantitative scoring system for the measurement of erosions as follows: normal: $<2 \mathrm{~mm}$; small erosion: $2 \mathrm{~mm}$; moderate erosion: > 2-<4 mm; large erosion: _ $4 \mathrm{~mm}^{23}$

Another semiquantitative scoring system is developed, where bone erosions are defined as follows: grade 0: normal bone surface; grade 1: bone surface irregularity without the defect being seen in two planes; grade 2: defect of the surface in two planes; grade 3: bone defect creating extensive bone destruction. ${ }^{11}$

\section{Cartilage}

US assessment can provide detailed imaging of the hyaline cartilage, identifying small cartilage abnormalities in patients affected by $\mathrm{RA}^{24}$, especially when dynamic US is performed as e.g. flexion of the finger joints and extension.US evaluation allows a reliable and valid measurement of cartilage at finger joints level, with great sensitivity compared to X-ray ${ }^{25}$.

\section{Effusion}

Joint effusion is a nonspecific feature of a large number of disease processes and readily identified at ultrasound. Fluid in a joint generally appears anechoic, but occasionally effusions appear more complex ${ }^{2}$.

Effusion is defined in ultrasound as an abnormal hypoechoic or anechoic intra-articular material that is displaceable and compressible, but it does not exhibit Doppler signal $^{26}$. 
Effusion is semiquantitatively scored as follows: grade 0: no effusion; grade 1: minimal amount; grade 2: moderate (without distension of the joint capsule); grade 3: extensive (with distension of the joint capsule) ${ }^{11}$.

\section{Power Doppler US}

Semiquantitative grading of the PD evaluation was as follows: grade 0: no flow; grade 1: singlevessel signals; grade 2: less than half of the area of the synovium filled with vessels; grade 3: more than half of the area of the synovium filled with vessels ${ }^{11}$. Figure $1 \mathrm{~A}-\mathrm{F}$

\section{New Horizons \\ Sonoelastography}

Sonoelastography (SEL) is a relatively new technique that can assess the elastic properties of tissues. SEL is based on the principle that the compression of tissue produces strain (displacement). SEL displays images related to a broad range of parameters that describe the spatial and temporal variations of tissue elasticity by processing time-varying echo data of an induced tissue displacement or strain ${ }^{27}$.

Several sonoelastographic methods are commercially available. The appropriate method depends on the stress application. In addition to compression elastography, there is shear wave elastography, transient elastography, and acoustic force elastography ${ }^{28}$, however, still this method has several limitations the most important is pressure on the skin with the freehand technique.

\section{D Ultrasound}

Three-dimensional ultrasound allows collection of data from a volume of tissue that can then be reconstructed and manipulated offline. This function potentially eliminates a degree of operator dependency that has otherwise been one of the potential disadvantages of US compared with MRI. Early studies with small patient numbers suggest that $3 \mathrm{D}$ imaging may improve the detection and reliability of US in detecting inflammatory and destructive changes in RA of the wrist and hand ${ }^{29}$, however resolution is less than obtained by conventional US and not adding more information as obtained by $2 \mathrm{D}$ ultrasound. Contrast enhanced ultrasound US contrast media, in comparison to PDUS, add important information at the angiogenetic level not only for early detection but also for follow-up and furthermore, allows a better visualization of disease activity in RA. However, the routine use of contrast agents for US examinations is not established enough as it is for MRI in this topic. The main obstacles in using US contrast media are higher costs (than B-mode US), technical limitations (for instance in near fields), the need for optimally designed bubbles for near field investigation at higher frequencies and a relatively short time window for examination. In addition, grading systems including synovial activity, which can be obtained by sensitive US assessment of synovial vascularity are necessary ${ }^{30-32}$.

\section{Current Progress}

The new seven-joint ultrasound (US7) score was proposed by Backhaus et al. This is the first US composite scoring system, combining soft tissue lesions (synovitis and tenosynovitis/paratenonitis) and destructive processes (erosions) in a single scoring system. The US7 score includes US examination of the following joints of the clinically more affected side: wrist, MCP II and III, PIP II and III, MTP II and $\mathrm{V}$. The joints are examined by GSUS and PDUS for synovitis and tenosynovitis/paratenonitis from a dorsal and palmar/plantar aspect, and for erosions from a dorsal, palmar/plantar and radial/lateral (only MCP II and MTP V) aspect ${ }^{33}$.

Accurate assessment of disease activity and joint damage in RAis important for monitoring treatment efficiency and for prediction of the outcome of the disease. Therefore, a reliable imaging method needs to be used. MSK-US is a sensitive method for the detection of both early inflammatory soft tissue lesions (eg, synovitis, tenosynovitis, and bursitis) and early bone lesions (e.g., erosions) in arthritic joint diseases and correlates well with MRI. Several musculoskeletal US scores are used for monitoring RA disease activity. Different qualitative $(0 / 1)$ and semiquantitative (0-3) systems and quantitative measurements are used ${ }^{11}$.

Despite these attractive features, the technique is still considered examiner-dependent and machine dependent. This opinion is based mainly on the fact that both acquisition and interpretation of US images determine the metric properties. Over the past decade, OMERACTultrasound Task Force, a group of interested international sonographers, has worked to address the metric qualities of musculoskeletal ultrasound in $\mathrm{RA}^{13}$.

The implementation of musculoskeletal US as a patient-friendly, reliable, bedside method in daily rheumatological practice is helpful and is essential for the objective examination of joints assumed to be affected. The use of a representative US sum/composite score, in which mostly active joint regions are included, reduces examination time and, at the same time, reflects a patient's overall disease activity ${ }^{11}$.

\section{Conclusion}

MSK-US is fastly progressing to be first although not the sole imaging modality tool for not only early diagnosis, but also therapeutic follow up, and monitoring the progress or remission of rheumatoid arthritis. New technologies as contrast media and sonoelastography are further expected to expand the diagnostic horizon. 

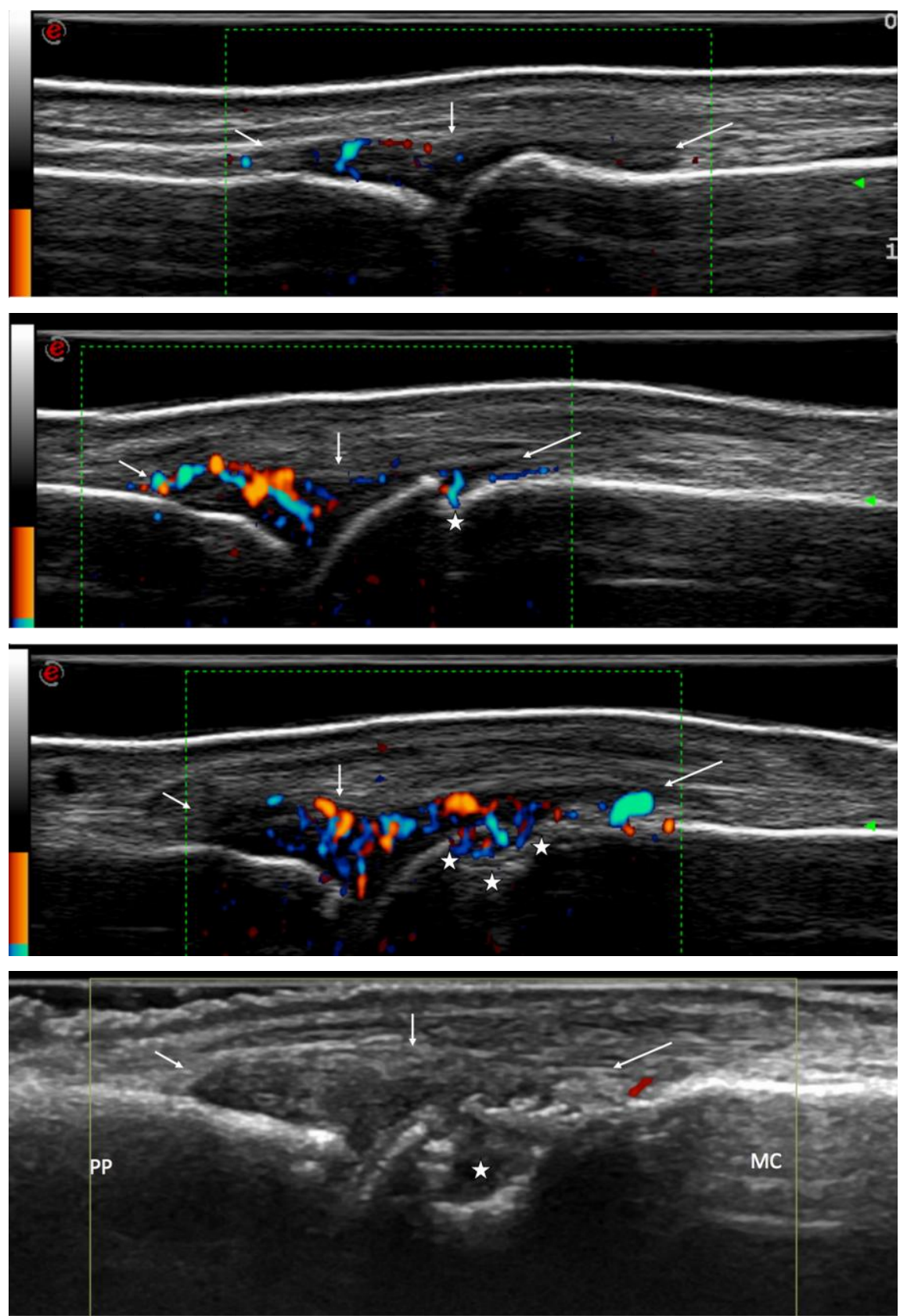

Figure 1. Sonography of finger joints. (a) Dorsal longitudinal plane PIP 3 with joint thickening, shows hypoechoic joint thickening (between arrows), capsular thickening, mild hyperemia, no erosions: this is an unspecific finding, can be seen in very early RA, but also in overuse or after trauma (PIP proximal interphalangeal joint). (b) Dorsal longitudinal plane MCP 2 with joint thickening, shows hypoechoic joint thickening (between arrows), capsular thickening, moderate hyperemia, questionable only small erosion (star), measuring less than $1 \mathrm{~mm}$, what can be seen in very early erosive status, differential diagnosis: feeding vessel (MCP Metacarpophalangeal joint). (c) Dorsal longitudinal plane MC3 2 with synovial proliferation, shows hypoechoic joint synovitis (between arrows), moderate to severe hyperemia, highly vascularized erosion (stars), measuring more than $2 \mathrm{~mm}$, what can be seen in erosive RA, negative in X ray (not shown). (d) Dorsal longitudinal plane PIP 2 with synovial proliferation, shows hypoechoic joint synovitis (between arrows), old huge avascular erosion (star), what can be seen also in X ray (not shown). Sonography gives the information of disease in remission, no hypervascularity found in the pannus nor in the erosion. $\mathrm{PP}=$ proximal phalanx, $\mathrm{MC}=$ metacarpal bone. 


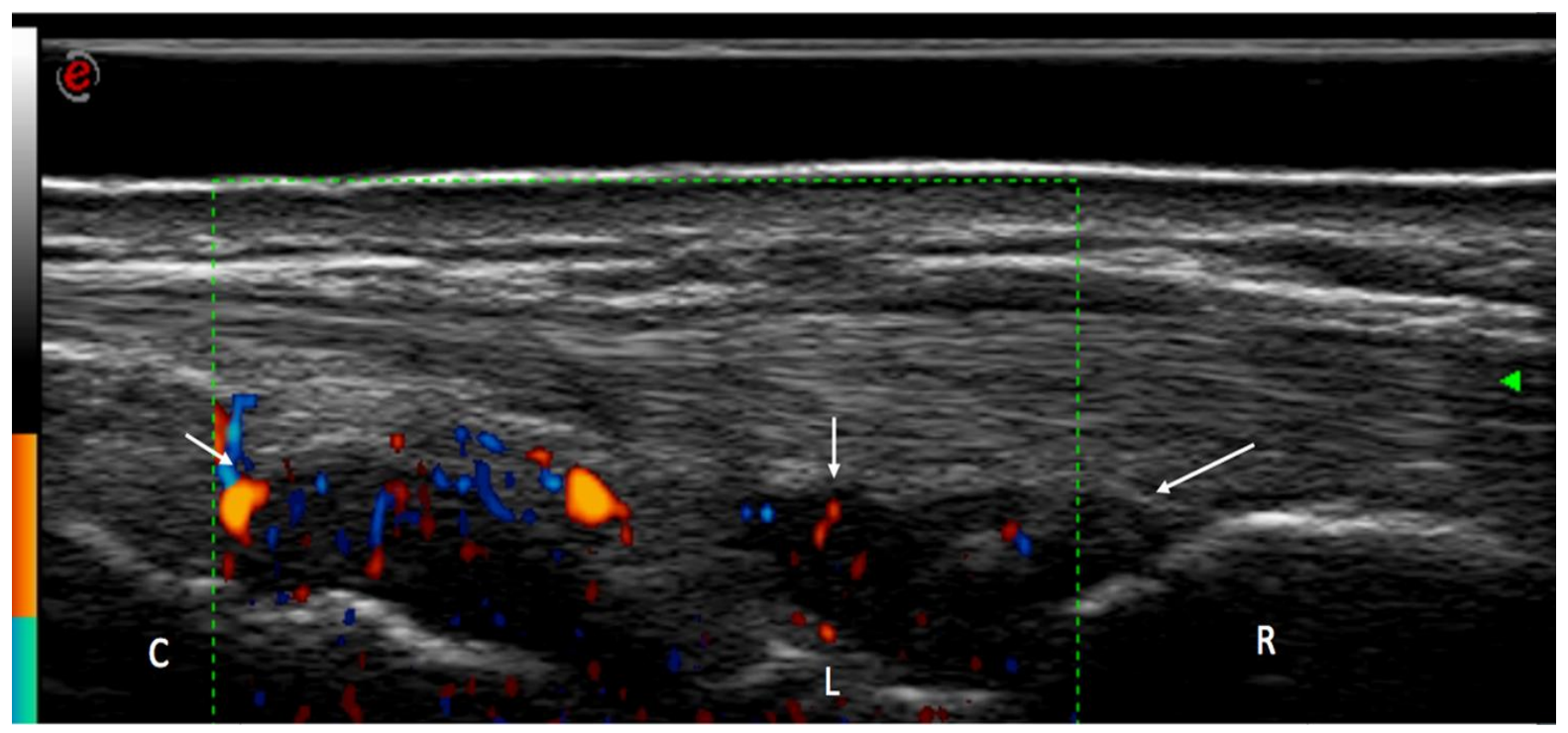

(a) Dorsal longitudinal at the wrist showing hypoechoic joint thickening at the radiocarpal and intercarpal joints (between arrows), mild hyperemia, no erosions ( $\mathrm{R}=$ Radius, $\mathrm{C}=$ capitatum, $\mathrm{L}=$ lunatum).

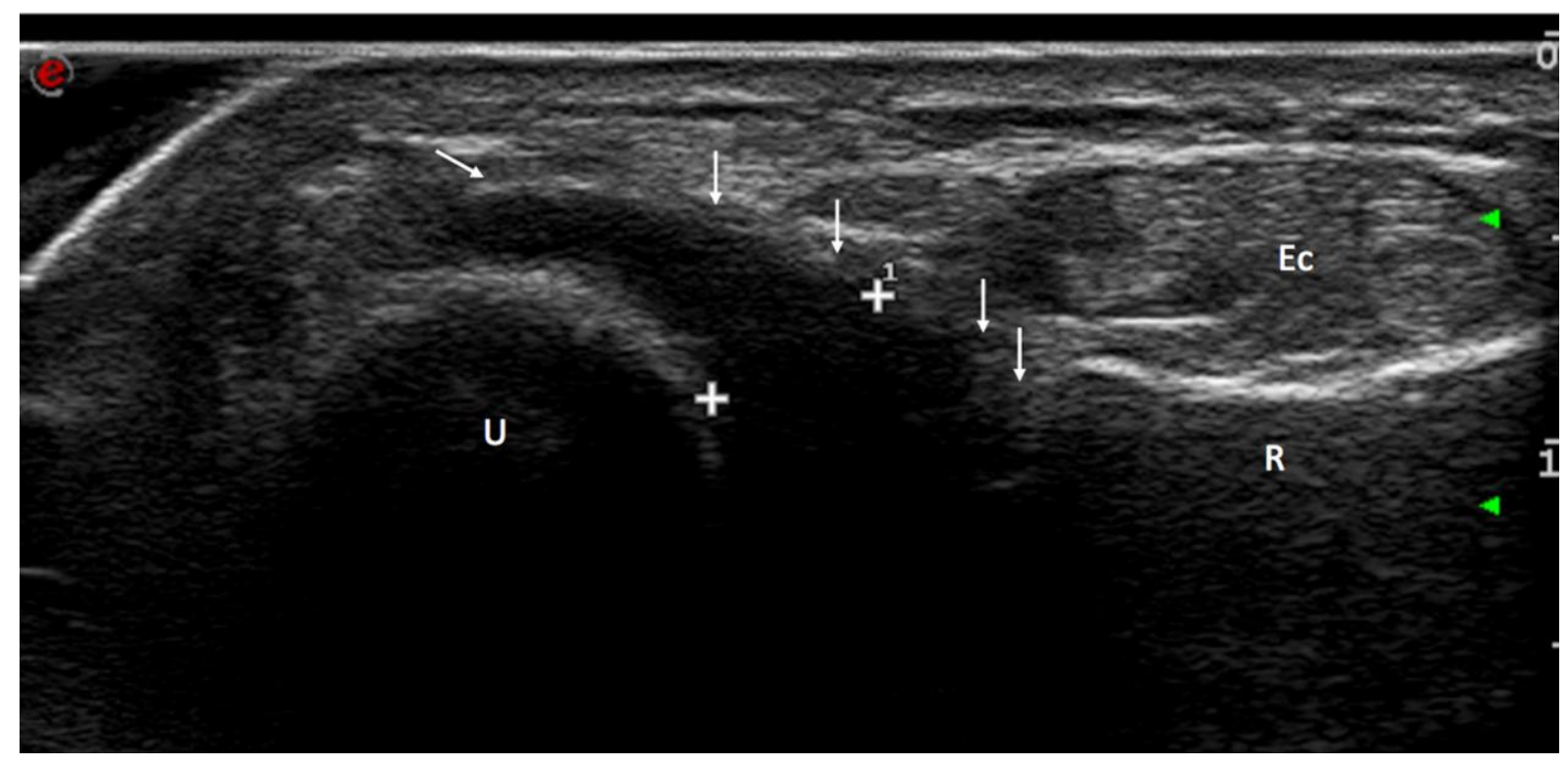

(b) Dorsal axial plane at the distal radioulnar joint with clear joint thickening, (between arrows), characteristically seen in $\mathrm{RA}$ affecting the distal radioulnar ( $\mathrm{R}=$ Radius, $\mathrm{U}=\mathrm{ulna}), \mathrm{Ed}=$ Extensor tendons).

Figure 2. Sonography of the wrist. 


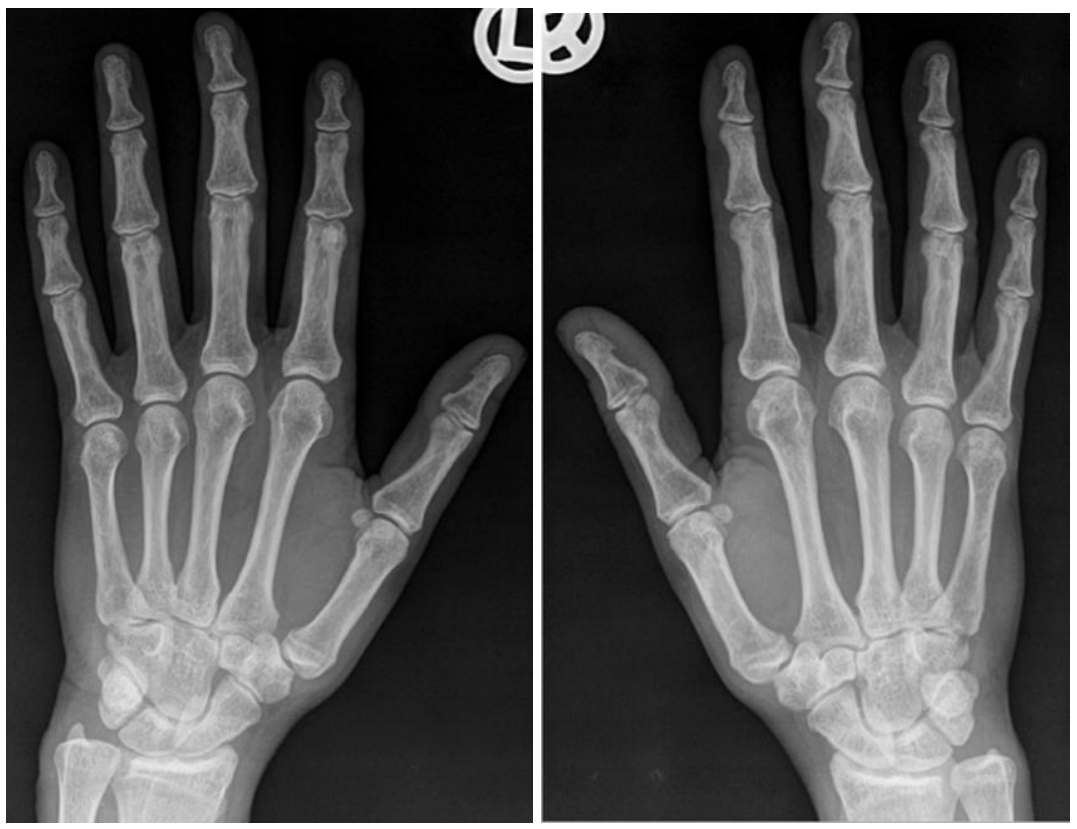

(a) X-ray of both hands: signs of osteoarthritis, no signs of rheumatoid arthritis.

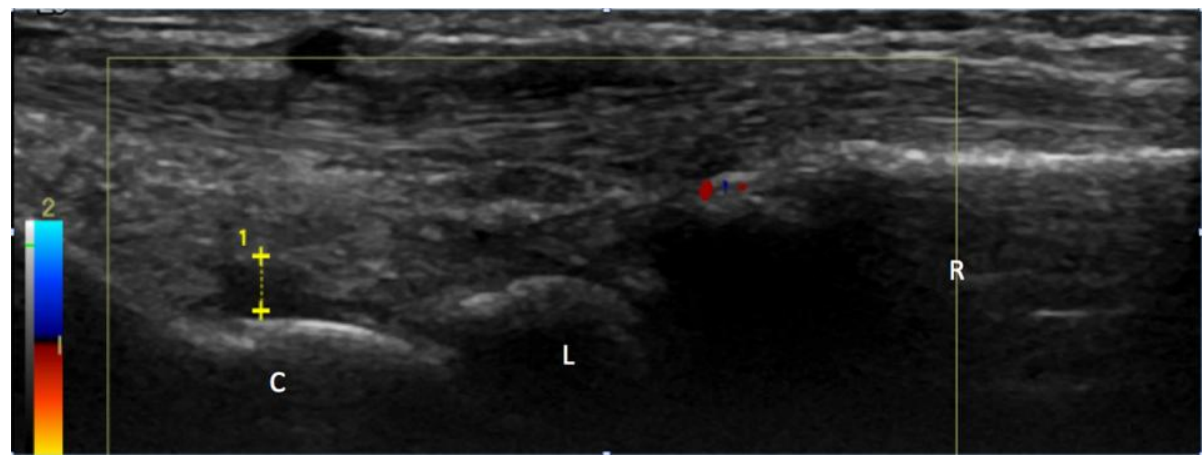

(b) Dorsal longitudinal at the right wrist showing discrete hypoechoic joint thickening at the radiocarpal and intercarpal joints (between arrows), no hyperemia, no erosions ( $\mathrm{R}=$ Radius, $\mathrm{C}=$ capitatum, $\mathrm{L}=$ lunatum). Unspecific finding.

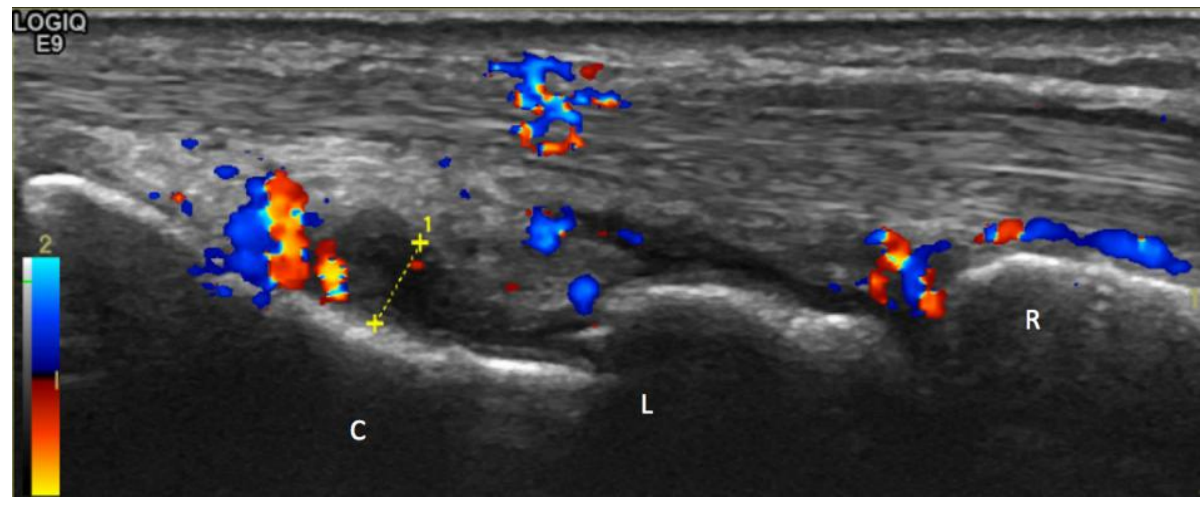

(c) Dorsal longitudinal at the left wrist showing distinct hypoechoic joint thickening at the radiocarpal and intercarpal joints (between arrows), moderate hyperemia, no erosions ( $\mathrm{R}=$ Radius, $\mathrm{C}=$ capitatum, $\mathrm{L}=$ lunatum). 


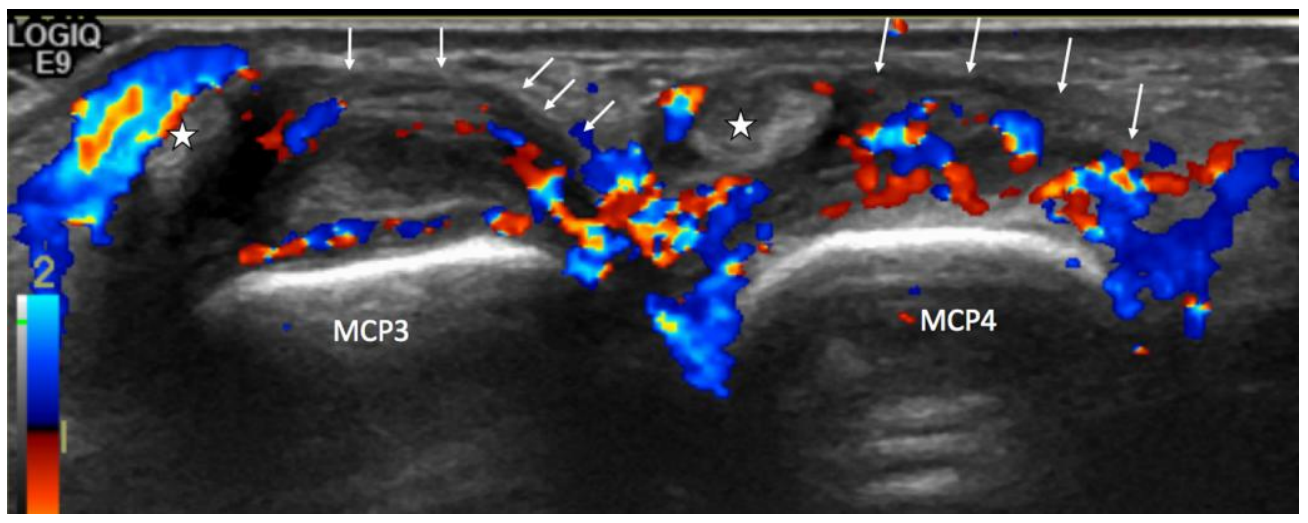

(d) Dorsal axial scan at the 3th and 4th MCP level, showing extensive hyperemia around extensor tendons, sagittal bands and intraarticular. Note: subluxation of the extensor tendons (star) because of elongation and partial rupture of the sagittal bands (arrows).

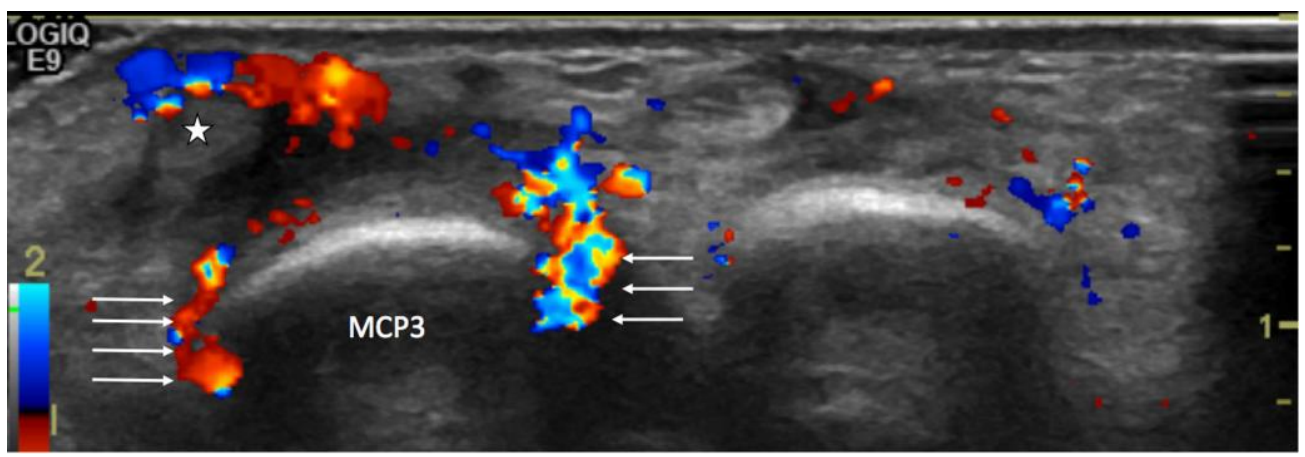

(e) Dorsal axial scan at the 3th MCP level, showing extensive hyperemia also towards the bare areas (arrows), where erosions can develope, at this time no erosions.

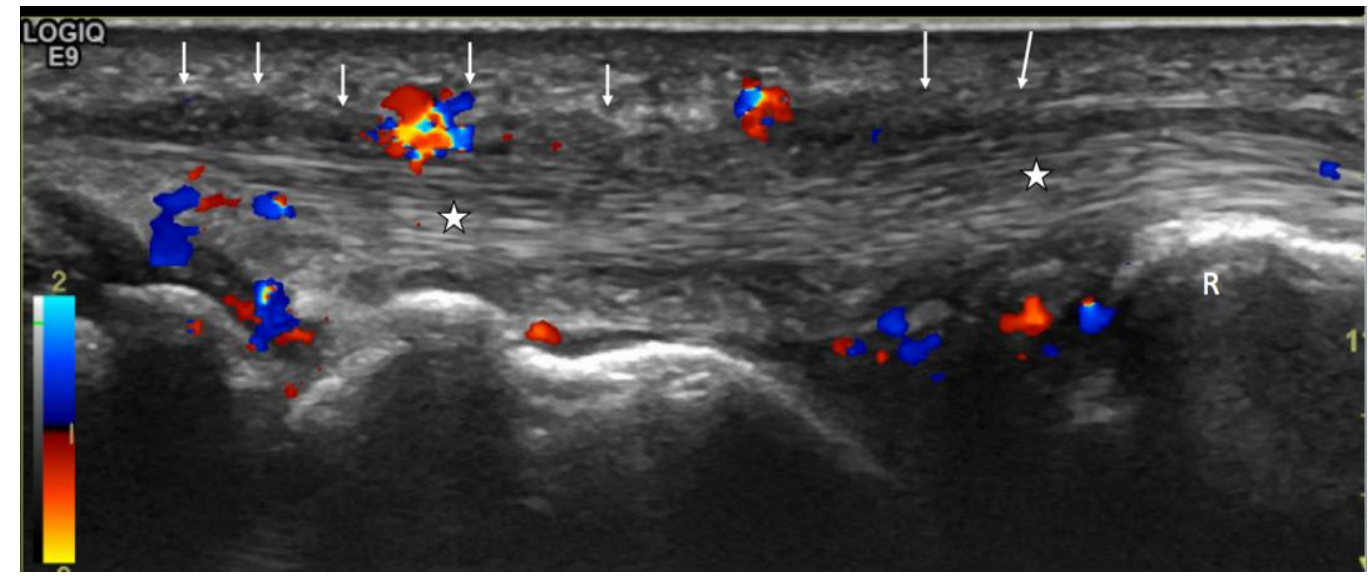

(f) Longitudinal scan over the ECU tendons (stars), showing hyperemia and synovial proliferation (arrows), a classical finding for RA.

Figure 3. Clinical case: 57 yr old female: late onset RA?

Summary: Because of symmetrical involvement of MCP joints, both ECU tendons and left radiocarpal joint the diagnosis of a late onset RA can be made, even no erosions at the moment are present. 


\section{REFERENGES}

1. Da Mota LM, Laurindo IM, dos Santos Neto LL, Lima FA, Viana SL, Mendlovitz PS, Fernandes JL. Imaging diagnosis of early rheumatoid arthritis. Rev Bras Reumatol. 2012 Oct;52(5):757-66. PMID: 23090375

2. Grainger AJ, Rowbotham EL. Rheumatoid arthritis. Semin Musculoskelet Radiol. 2013 Feb;17(1):69-73. doi: 10.1055/s-0033-1333939. PMID: 23487337

3. Janta I, Naredo E, Martínez-Estupiñán L, Nieto JC, De la Torre I, Valor L, Estopiñán L, Bello N, Hinojosa M, González CM, López-Longo J, Monteagudo I, Montoro M, Carreño L. Patient selfassessment and physician's assessment of rheumatoid arthritis activity: which is more realistic in remission status? A comparison with ultrasonography. Rheumatology (Oxford). 2013 Sep 17. PMID:24046468.

4. Rizzo C, Ceccarelli F, Gattamelata A, Vavala C, Valesini G, Iagnocco A. Ultrasound in rheumatoid arthritis. Med Ultrason. 2013 Sep;15(3):199-208. PMID: 23979615

5. Ohrndorf S, Backhaus M. Musculoskeletal ultrasonography in patients with rheumatoid arthritis. Nat Rev Rheumatol. 2013 Jul;9(7):433-7. PMID: 23689232

6. Hartung W, Backhaus M, Ohrndorf S. [Joint sonography in rheumatology]. Z Rheumatol. 2013 Oct;72(8):791-803. doi: 10.1007/s00393-013-12328. German. PMID: 24085531

7. Arend CF. Ultrasonography in rheumatoid arthritis: what rheumatologists should know. Rev Bras Reumatol. 2013 Feb;53(1):88-100. PMID: 23588519

8. Gärtner M, Mandl P, Radner H, Supp G, Machold KP, Aletaha D, Smolen JS. Sonographic joint assessment in rheumatoid arthritis: associations with clinical joint assessment during a state of remission. Arthritis Rheum. 2013 Aug;65(8):200514. doi: 10.1002/art.38016.PMID:23686535

9. M Backhaus, G-R Burmester, $\mathrm{T}$ Gerber, et al. Guidelines for musculoskeletal ultrasound in rheumatology. Ann Rheum Dis 2001 60: 641-649.

10. Richard J Wakefield, Maria Antonietta D'Agostino, Esperanza Naredo, et al. Ultrasound initiative improve RA outcomes? After treat-to-target: can a targeted. Ann Rheum Dis 2012, 71: 799-803. doi: 10.1136/annrheumdis-2011-201048, PMID: 22562979

11. Ohrndorf S, Backhaus M. Advances in sonographic scoring of rheumatoid arthritis. Ann Rheum Dis. 2013 Apr;72 Suppl 2:ii69-75. doi: 10.1136/annrheumdis2012-202197. PMID: 23466958

12. D'Agostino MA, Conaghan PG, Naredo E, et al. The OMERACT ultrasound task force-advances and priorities. J Rheum 2009;36:1829-32. PMID: 19671821
13. Bruyn GA, Hanova P, Iagnocco A, d'Agostino MA, Möller I, Terslev L, Backhaus M, Balint PV, Filippucci E, Baudoin P, van Vugt R, Pineda C, Wakefield R, Garrido J, Pecha O, Naredo E; on behalf of the OMERACT Ultrasound Task Force. Ultrasound definition of tendon damage in patients with rheumatoid arthritis. Results of a OMERACT consensusbased ultrasound score focussing on the diagnostic reliability. Ann Rheum Dis. 2013 Aug 22. doi:10.1136/annrheumdis-2013-203596 PMID: 23940212

14. Wakefield RJ, Balint PV, Szkudlarek M, et al. Musculoskeletal ultrasound including definitions for ultrasonographic pathology. J Rheum 2005;32:2485-7. PMID: 16331793

15. Szudlarek M, Court-Payen M, Jacobsen S, Klarlund M, Thomsen HS, Ostergaard M. Interobserver agreement in ultrasonography of the finger and toe joints in rheumatoid arthritis. Arthritis Rheum 2003; 48(4):955-62. PMID: 12687537

16. Weidekamm C, Koller M, Weber M, Keinberger F. Diagnostic value of high resolution $\mathrm{B}$ mode and Doppler sonography for imaging of hand and finger joints in rheumatoid arthritis. Arthritis Rheum 2003; 48(2):325-33. PMID: 12571840

17. Naredo E, Möller I, Cruz A, Carmona L, Garrido J. Power Doppler ultrasonographic monitoring of response to antitumor necrosis factor therapy in patients with rheumatoid arthritis. Arthritis Rheum 2008; 58: 2248-2256. doi: 10.1002/art.23682. PMID: 18668537

18. Koski JM, Saarakkala S, Helle M, Hakulinen U, Heikkinen JO, Hermunen H. Power Doppler ultrasonography and synovitis: correlating ultrasound imaging with histopathological findings and evaluating the performance of ultrasound equipment. Ann Rheum Dis 2006;65(12):15901595. PMID: 16707536

19. Torp-Pedersen ST, Terslev L. Settings and artefacts relevant in colour/power Doppler ultrasound in rheumatology. Ann Rheum Dis 2008;67(2):143149. PMID: 18055471

20. Jain A, Nanchahal J, Troeberg L, Green P, Brennan F. Production of cytokines, vascular endothelial growth factor, matrix metalloproteinases, and tissue inhibitor of metalloproteinases by tenosynovium demonstrates its potential for tendon destruction in rheumatoid arthritis. Arthritis Rheum 2001; 44: 1754-1760. PMID: 11508425

21. Vlad V, Berghea F, Libianu S, et al. Ultrasound in rheumatoid arthritis - volar versus dorsal synovitis evaluation and scoring. BMC Musculoskelet Disord 2011;12:124. doi: 10.1186/1471-2474-12-124. PMID: 21635793

22. Farrant JM, Grainger AJ, O'Connor PJ. Advanced imaging in rheumatoid arthritis. Part 2. Erosions. Skeletal Radiol 2007; 36(5):381-9. PMID: 17091308 
23. Wakefield RJ, Gibbon WW, Conaghan PG, et al. The value of sonography in the detection of bone erosions in patients with rheumatoid arthritis: a comparison with conventional radiography. Arthritis Rheum 2000;43:2762-70. PMID: 11145034

24. Disler DG, Raymond E, May DA, Wayne JS, McCauley TR. Articular cartilage defects: in vitro evaluation of accuracy and inter-observer reliability for detection and grading with US. Radiology 2000; 215: 846-851. PMID: 10831709

25. Möller B, Bonel H, Rotzetter M, Villiger PM, Ziswiler HR. Measuring finger joint cartilage by ultrasound as a promising alternative to conventional radiograph imaging. Arthritis Rheum 2009; 61: 435-41. doi: 10.1002/art.24424. PMID: 19333990

26. Chakr RM, Mendonça JA, Brenol CV, Xavier RM, Brenol JC. Assessing rheumatoid arthritis disease activity with ultrasound. Clin Rheumatol. 2013 Sep;32(9):1249-54. doi: 10.1007/s10067-013-22916 PMID: 23700041

27. Klauser AS, Faschingbauer R, Jaschke WR. Is sonoelastography of value in assessing tendons? Semin Musculoskelet Radiol. 2010 Sep;14(3):32333. doi: 10.1055/s-0030-1254521 PMID: 20539957

28. Klauser AS, Miyamoto H, Tamegger M, Faschingbauer R, Moriggl B, Klima G, Feuchtner GM, Kastlunger M, Jaschke WR. Achilles tendon assessed with sonoelastography: histologic agreement. Radiology. 2013 Jun;267(3):837-42. doi: 10.1148/radiol.13121936 PMID: 23449953

29. Naredo E, Möller I, Acebes C, et al. Threedimensional volumetric ultrasonography. Does it improve reliability of musculoskeletal ultrasound? Clin Exp Rheumatol 2010;28(1):79-82. doi: 10.3899 PMID: 23322469
30. Klauser A, Demharter J, De Marchi A, Sureda D, Barile A, Masciocchi C, Faletti C, Schirmer M, Kleffel T, Bohndorf K; IACUS study group. Contrast enhanced gray-scale sonography in assessment of joint vascularity in rheumatoid arthritis: results from the IACUS study group. ur Radiol. 2005 Dec;15(12):2404-10. Epub 2005 Aug 16.

31. Klauser AS, Franz M, Arora R, Feuchtner GM, Gruber J, Schirmer M, Jaschke WR, Gabl MF. Detection of vascularity in wrist tenosynovitis: power doppler ultrasound compared with contrastenhanced grey-scale ultrasound. Arthritis Res Ther. 2010;12(6):R209. doi: 10.1186/ar3185. Epub 2010 Nov 9.

32. Klauser A, Frauscher F, Schirmer M, Halpern E, Pallwein L, Herold M, Helweg G, ZurNedden D. The value of contrast-enhanced color Doppler ultrasound in the detection of vascularization of finger joints in patients with rheumatoid arthritis. Arthritis Rheum. 2002 Mar;46(3):647-53.

33. Backhaus $\mathrm{M}$, Ohrndorf $\mathrm{S}$, Kellner $\mathrm{H}$, et al. Evaluation of a novel 7-joint ultrasound score in daily rheumatologic practice: a pilot project. Arthritis Rheum 2009;61:1194-201.doi: 10.1002/art.24646. PMID: 19714611

\section{Core Tip [summary]}

This review describes the role of musculoskeletal ultrasound in the diagnosis of rheumatoid arthritis, with special concentration on its effect on treatment monitoring, and on disease prognosis. Highlight is made on ultrasound assessment of the synovium, effusion, tendon disease together with the role of power Doppler is made. Special emphasis is given to new horizons like contrast enhanced ultrasound, elastography and three dimensional ultrasound. 